\title{
A translational exploration of the effects of WNT2 variants on altered cortical structures in autism spectrum disorder
}

\author{
Yi-Ling Chien, MD, PhD; Yu-Chieh Chen, MS; Yen-Nan Chiu, MD; \\ Wen-Che Tsai, MD, PhD; Susan Shur-Fen Gau, MD, PhD
}

\begin{abstract}
Background: Evidence suggests that cortical anatomy may be aytpical in autism spectrum disorder. The wingless-type MMTV integration site family, member 2 (WNT2), a candidate gene for autism spectrum disorder, may regulate cortical development. However, it is unclear whether WNT2 variants are associated with altered cortical thickness in autism spectrum disorder. Methods: In a sample of 118 people with autism spectrum disorder and 122 typically developing controls, we investigated cortical thickness using FreeSurfer software. We then examined the main effects of the WNT2 variants and the interactions of group $\times$ SNP and age $\times$ SNP for each hemisphere and brain region that was altered in people with autism spectrum disorder. Results: Compared to neurotypical controls, people with autism spectrum disorder showed reduced mean cortical thickness in both hemispheres and 9 cortical regions after false discovery rate correction, including the right cingulate gyrus, the orbital gyrus, the insula, the inferior frontal gyrus (orbital part and triangular part), the lateral occipitotemporal gyrus, the posterior transverse collateral sulcus, the lateral sulcus and the superior temporal sulcus. In the full sample, 2 SNPs of WNT2 (rs6950765 and rs2896218) showed age $\times$ SNP interactions for the mean cortical thickness of both hemispheres, the middle-posterior cingulate cortex and the superior temporal cortex. Limitations: We examined the genetic effect for each hemisphere and the 9 regions that were altered in autism spectrum disorder. The age effect we found in this cross-sectional study needs to be examined in longitudinal studies. Conclusion: Based on neuroimaging and genetic data, our findings suggest that WNT2 variants might be associated with altered cortical thickness in autism spectrum disorder. Whether and how these WNT2 variants might involve cortical thinning requires further investigation. Trial registration: ClinicalTrials.gov no. NCT01582256. Protocol registration: National Institutes of Health no. NCT00494754.
\end{abstract}

\section{Introduction}

Atypical age-related changes in cortical anatomy have been reported frequently in people with autism spectrum disorder, ${ }^{1,2}$ presenting as early cortical overgrowth followed by cortical dysmaturation throughout childhood and adolescence, suggesting exaggerated or slowed thinning in different cortical regions in youth., Early longitudinal studies identified several cortical regions that showed deviated trajectories in people with autism spectrum disorder (aged 3-36 yr, mean $16.8 \mathrm{yr}$ ) compared to typically developing controls, including the parietal and occipital cortices., 5 Yang and colleagues ${ }^{7}$ also found a lack of normative age-related cortical thinning in regions related to the social perception, language, selfreferential and action observation networks in preadolescents with autism spectrum disorder (aged 4-12 yr, mean $8.4 \mathrm{yr}$ ), further supporting the hypothesis that cortical development in autism spectrum disorder undergoes accelerated expansion in early childhood and altered thinning in adolescence and early adulthood. ${ }^{3}$ A recent study ${ }^{1}$ found atypical agerelated changes in cortical thickness in people with autism spectrum disorder (aged 6-30 yr), primarily in frontal and temporoparietal areas associated with the symptoms of autism spectrum disorder, and these differentiated autism spectrum disorder from typical development.

A large-scale, multisite study $(n=1327 \text {, aged } 2-65 \mathrm{yr})^{8}$ identified greater cortical thickness (including the superior temporal gyrus and inferior frontal sulcus) in people with autism spectrum disorder $(n=491)$. The study also found subtle but important age-specific cortical alterations: greater thickness in

Correspondence to: S.S. Gau, Department of Psychiatry, National Taiwan University Hospital and College of Medicine, No.7, Chung-Shan South Rd., Taipei 10002, Taiwan; gaushufe@ ntu.edu.tw

Submitted Jan. 26, 2021; Revised Jun. 19, 2021; Accepted Jul. 28, 2021

Cite as: J Psychiatry Neurosci 2021 December 3;46(6). doi: 10.1503/jpn.210022 
younger children with autism spectrum disorder (6-14 yr) in the lateral temporal and frontal regions and in the posterior cingulate cortex. Another study, ${ }^{2}$ analyzing an ENIGMA data set $(n=1571$, mean age \pm standard deviation [SD] $15.4 \pm 8.6 \mathrm{yr})$, found a negative linear relationship between age and cortical thickness in several brain regions and a quadratic age effect in the insula, indicating that people with autism spectrum disorder show a peak of difference in cortical thickness around adolescence, with greater thickness in the frontal cortex and less thickness in the temporal cortex. Both cross-sectional largescale studies support the concept of age-related alterations in cortical thickness in people with autism spectrum disorder.

Atypical brain development may be regulated or moderated by genetic mechanisms. The brain cortex can be parcellated into clusters of maximal shared genetic influence based on genetic correlations between brain regions, ${ }^{9,10}$ supporting the hypothesis that genetically programmed neurodevelopmental events may affect the organization of the cerebral cortex decades later. ${ }^{9,11,12}$ As well, Fjell and colleagues ${ }^{13}$ found that both developmental and adult age-related changes in cortical thickness closely followed the genetic organization of the cerebral cortex: change rates varied as a function of the genetic similarity between regions. However, whether the candidate genes that regulate central nervous system patterning are also associated with cortical thickness in neurodevelopmental disorders such as autism has received little attention compared to other indexes of brain structure, such as brain volume ${ }^{14,15}$ or structural connectivity. ${ }^{16,17}$ Cortical thickness is related to the migration of neurons and the organization of minicolumns, ${ }^{18}$ and it may represent dendritic arborization and pruning in the grey matter. ${ }^{19}$

The wingless-type MMTV integration site family member 2 (WNT2) gene is a candidate gene for autism spectrum disorder that is essential for regulating early cortical development by promoting cortical dendrite growth and spine formation. ${ }^{20-22}$ The Wnt signalling pathway controls the proliferation of progenitor cells; ${ }^{23}$ inhibition of Wnt signalling disrupts dendritic spine development and reduces the size and complexity of dendritic arborization..$^{22}$ In an animal model of autism spectrum disorder using prenatal valproate exposure that causes stereotypy and decreased social behaviours in rodents, ${ }^{24}$ the gene expression and translation of Wnt2 (and genes under transcriptional control of the Wnt pathway) were upregulated in the prefrontal cortex and hippocampus, ${ }^{25}$ implying the dysregulation of Wnt2 expression in specific brain areas in autism spectrum disorder.

In humans, WNT2 variants have been associated with several neurodevelopmental disorders, including autism spectrum disorder. ${ }^{26-28}$ Wassink and colleagues ${ }^{28}$ screened the WNT2 coding sequence for mutations and found 2 families containing nonconservative coding-sequence variants that segregated with autism. They also identified linkage disequilibrium with a single nucleotide polymorphism (SNP) from the 3'-untranslated region of WNT2 in autism-affected sibling-pair families with severe language abnormalities. ${ }^{28}$ However, the association of that SNP with autism spectrum disorder was not replicated in an independent data set of 135 singletons and 82 multiplex families. ${ }^{29}$ Marui and colleagues ${ }^{27}$ reported significant associations between autism and 3 SNPs of WNT2 (rs3779547, rs4727847 and rs3729629), or 2 major haplotypes (A-T-C and GG-G, consisting of rs3779547, rs4727847 and rs3729629 in intron 4) in a case-control analysis (170 people with autism v. 214 controls) and a transmission disequilibrium test analysis (98 autism trios). Furthermore, our previous studies have shown that the WNT2 haplotypes composed of rs2896218 and rs6950765 (G-G; 2 SNPs in intron 4) were not only associated with the risk of autism spectrum disorder, ${ }^{26}$ but may also moderate clinical phenotypes, such as age at first phrase (SNP rs2896218 or a 3-locus haplotype [A-G-A, rs4727845, rs2228946 and rs2896218]) and several symptom domains (e.g., rs2896218rs6950765 [G-G] v. more severe stereotyped behaviours). ${ }^{30}$

The WNT genes encode secreted growth factor-like proteins that involve growth regulation, differentiation, dendritic spine development and arborization, ${ }^{22}$ which may contribute to cortical thickness. ${ }^{19}$ Given the regulatory role of WNT2 in cortical development, the potential association between WNT2 variants and cortical thickness and thinning over time is of particular interest, specifically for the cortical regions implicated in neurodevelopmental disorders such as autism spectrum disorder.

This study aimed to investigate the genetic effect of WNT2 on cortical thickness in regions that are altered in people with autism spectrum disorder, and to examine whether these genetic effects varied between people with autism spectrum disorder and typically developing controls in an age-dependent manner. Given that WNT2 variants have been genetically associated with autism spectrum disorder and its clinical severity, and that they may regulate cortical development, we hypothesized that the genetic variants of WNT2 previously reported (i.e., rs2896218 and rs6950765) might be associated with altered cortical thickness or thinning in autism spectrum disorder.

\section{Methods}

\section{Participants}

We recruited 118 people with autism spectrum disorder (age: range 6-28 yr, mean \pm SD $13.1 \pm 4.6 \mathrm{yr}$, median $14 \mathrm{yr}$; male: $n=113,95.8 \%$ ) from National Taiwan University Hospital, Taipei, Taiwan. We also recruited 122 typically developing controls (age: range 7-56 yr, mean \pm SD $21.0 \pm$ $9.7 \mathrm{yr}$, median $19 \mathrm{yr}$; male: $n=75,61.5 \%$ ) through school referral or advertisements.

All participants with autism spectrum disorder were diagnosed by senior board-certified child psychiatrists based on DSM-5 diagnostic criteria; ${ }^{31}$ clinical diagnosis was further confirmed in structural interviews using the Chinese version of the Autism Diagnostic Interview-Revised (ADI-R). ${ }^{32}$ All typically developing controls were clinically evaluated to rule out any traits of autism; their parents were interviewed using the Chinese version of the Kiddie Schedule for Affective Disorders and Schizophrenia, Epidemiological version (K-SADS-E), ${ }^{33}$ to exclude current or lifetime autism spectrum disorder and other major neuropsychiatric disorders (e.g., attention-deficit/hyperactivity disorder, schizophrenia, mood disorders, anxiety disorders or other neurodevelopmental disorders). We also collected a detailed family history for each participant to ensure that every first- or second-degree 
relative was Han Chinese and that no first- or second-degree relatives of the typically developing controls had a diagnosis of autism spectrum disorder. Participants with a full-scale IQ (FIQ) lower than 70 were excluded from the study. Participants with a systemic disease, chromosome deficit, any neurologic disorder or any major psychiatric disorder were also excluded. All participants were ethnic Han Chinese.

\section{Procedure}

The research ethics committee approved the study before its implementation (approval no. 201201006RIB; ClinicalTrials. gov no. NCT01582256). After the purposes and procedures of the study were fully explained and confidentiality was assured, we obtained written informed consent from the participants and their parents. All participants were clinically evaluated by experienced child psychiatrists, followed by ADI-R interviews with the parents (for autism spectrum disorder group only) and K-SADS-E interviews with the participants and parents (for all participants) to confirm the diagnosis of autism spectrum disorder and any other diagnostic status.

To determine IQ, all participants were assessed using the Wechsler Intelligence Scale for Children (version III) if they were younger than 16 years, or the Wechsler Adult Intelligence Scale (version IV) if they were older than 16 years. All participants completed a head MRI. We drew peripheral blood samples for genotyping. The parents also completed the Chinese version of the Social Responsiveness Scale (SRS), described below.

\section{Measures}

The ADI- $\mathrm{R}^{32}$ is a standardized, comprehensive, semistructured, investigator-based interview of the caregiver. It covers most developmental and behavioural aspects of autism spectrum disorder, including reciprocal social interaction and communication, restricted behaviours, repetitive behaviours and stereotyped behaviours for children from 18 months to adulthood. The Chinese version of the ADI-R was approved by the World Psychological Association in $2007^{34}$ and has been widely used in clinical studies to validate the diagnosis of autism spectrum disorder in study samples of ethnic Chinese populations.

The SRS, ${ }^{32}$ a widely used quantitative measure of autistic traits in the general population, is a 65-item rating scale that measures the severity of autism-spectrum symptoms in natural social settings over the preceding 6 months. In this study, caregivers used the SRS to report on the autistic traits of participants with autism spectrum disorder. The SRS has good reliability and validity. ${ }^{32}$ The Chinese version ${ }^{35}$ has a 4 -factor structure (i.e., social communication, stereotyped behaviours/ interest, social awareness and social emotion) but is better conceptualized as a 1-factor model. We used the SRS total score in our analyses as a general index of autism-like social deficits.

\section{SNP selection and genotyping}

We prepared genomic DNA from peripheral blood using the Puregene DNA purification system (Gentra Systems Inc.) according to the manufacturer's instructions. We selected 6 tag
SNPs of WNT2 (i.e., rs4727845, rs2228946, rs2896218, rs6950765, rs10227271 and rs2285545) based on the Ensembl-DAS Integration from the Center for Human Genetics ${ }^{36}$ and the International HapMap project (www.hapmap.org). ${ }^{26}$ We decided the number of linkage disequilibrium blocks according to the HapMap Genome Browser release database and selected 1 or 2 tag SNPs that represented each block spanning the whole gene, including the 5'- and 3'-untranslated regions. We designed the primers for the selected SNPs using a method detailed in a previous study. ${ }^{26}$ All SNP genotyping was performed in the SEQUENOM MassARRAY System using matrix-assisted laser desorption/ionization-time of flight mass spectrometry. During a pretest of the 6 selected SNPs, we discarded rs4727845 because of a concern about primer interaction on the iPLEX platform. Success rates for genotyping were mostly $99 \%$ to $100 \%$, except for rs10227271 (64\% 80\%), and rs2228946 had a minor allele frequency lower than 5\%. Therefore, these SNPs were also excluded from further analysis.

\section{MRI data acquisition}

We acquired images for all participants on a $3 \mathrm{~T}$ MRI system (Trio; Siemens) with a 32-channel head coil. Head movement was restricted with expandable foam cushions and was assessed immediately after image acquisition. We acquired high-resolution $T_{1}$-weighted MRIs covering the whole head with a 3-dimensional magnetization-prepared rapid gradient echo (MPRAGE) sequence (repetition time 52530 ms, echo time $3.4 \mathrm{~ms}$, flip angle $59^{\circ}$ ), resulting in an isotropic spatial resolution of $1 \mathrm{~mm}^{3}$.

\section{MRI data analysis: whole-brain segmentation and cortical thickness calculation}

For quality assurance, images with excessive in-scanner head motion were excluded based on the Human Connectome Project pipeline. ${ }^{37}$ We used FreeSurfer V5.2.0 (https://surfer. nmr.mgh.harvard.edu/) on a 64-bit Linux operating system to reconstruct the cortical surface from the MPRAGE images. ${ }^{38}$ We performed whole-brain volumetric segmentation..$^{39,40} \mathrm{Im}$ ages with a Euler number (a measure of the topological complexity of the reconstructed cortical surface calculated by the number of vertices minus the number of edges plus the number of faces) ${ }^{37}$ of exactly 2 (indicating adequate data quality) underwent further analysis. ${ }^{41}$ Then, we used the FreeSurfer automatic cortical parcellation routine to automatically identify and label cortical regions according to the Desikan atlas. ${ }^{42}$

Cortical thickness was automatically calculated by computing the shortest distance between the white matter boundary and the pial surface at each vertex. ${ }^{43}$ The reliability of the cortical thickness calculated by FreeSurfer has been assured. ${ }^{44}$ The automatic reconstruction and calculation were then reprocessed after manual correction for errors. In brief, these procedures included the removal of non-brain tissue; automated Talairach transformation; segmentation of subcortical white matter and deep grey matter volumetric structures; intensity normalization; tessellation of grey matter and white matter boundaries; automated topology correction; 
and surface deformation for optimal placement of the borders between grey matter and white matter, and between grey matter and cerebrospinal fluid. The automatic parcellation of cortical regions derived 74 brain regions in each hemisphere, and we then obtained the thickness of each region.

\section{Statistical analysis}

We used SAS (version 9.4; SAS Institute Inc.) to perform statistical analyses. We used analysis of variance to compare the means of age, IQ and SRS subscores between people with autism spectrum disorder and typically developing controls using a generalized linear model and the PROC GLM procedure.

We employed a 2-step approach to examine the effects of genetic variants on cortical thickness in regions that differed between people with autism spectrum disorder and typically developing controls. First, we identified cortical regions that were altered in autism spectrum disorder by comparing the cortical thickness of both hemispheres and 74 brain regions in an age- and sex-compatible subsample, in which typically developing controls with the same sex and the closest age $( \pm 1 \mathrm{yr}$ ) were selected for comparison with each participant with autism spectrum disorder aged 8-20 years. We controlled for sex, age, FIQ and intracranial volume (to correct for individual differences in head size $)^{7,45-47}$ in the comparison. We initially included the quadratic term of age in the model, but we eventually discarded it because the main results were not different. We applied false discovery rate (FDR) correction for multiple comparisons within each hemisphere (containing
74 regions). The mean cortical thickness of both hemispheres and the 9 regions that were significantly different in people with autism spectrum disorder in the age- and sex-compatible subsample were also significantly different in people with autism spectrum disorder for the full sample.

Using these results from the first step, we examined the effect of genetic variants for the full sample. For both hemispheres and the 9 regions that were altered in people with autism spectrum disorder, we conducted multivariate analyses to test the main effect of each SNP on cortical thickness, including diagnosis, sex, age, FIQ and intracranial volume in the model. We further tested whether the effect of SNP differed between the 2 groups by adding an interaction term $(\mathrm{SNP} \times$ diagnosis) to the model. Considering the age range of our sample, we also tested the interaction between SNP effect and age by examining another interaction term (SNP $\times$ age) in the full sample. We also tested the same model for participants with autism spectrum disorder and typically developing controls separately to investigate the SNP $\times$ age interaction specifically for those 2 groups.

\section{Results}

\section{Sample characteristics}

Participant IQ profiles were within the normal range and were significantly lower in people with autism spectrum disorder than in typically developing controls (Table 1). The ADI-R subscores for people with autism spectrum

Table 1: Participant demographic characteristics

\begin{tabular}{|c|c|c|c|c|}
\hline Characteristic & $\begin{array}{l}\text { Autism spectrum disorder* } \\
\qquad n=118\end{array}$ & $\begin{array}{l}\text { Typically developing controls* } \\
\qquad n=122\end{array}$ & $F / \chi^{2}$ & $p$ value \\
\hline Age, yr & $13.11 \pm 4.64$ & $20.98 \pm 9.70$ & $F=63.27$ & $<0.001$ \\
\hline Male, $n(\%)$ & $113(95.8)$ & $75(61.5)$ & $\chi^{2}=41.6$ & $<0.001$ \\
\hline Left-handedness, $n(\%)$ & $9(7.6)$ & $5(4.1)$ & $\chi^{2}=1.36$ & 0.24 \\
\hline Verbal IQ & $100.8 \pm 19.9$ & $111.6 \pm 10.9$ & $F=28.51$ & $<0.001$ \\
\hline Performance IQ & $99.7 \pm 21.1$ & $111.5 \pm 13.3$ & $F=26.99$ & $<0.001$ \\
\hline Full-scale IQ & $100.1 \pm 20.1$ & $112.3 \pm 11.7$ & $F=33.31$ & $<0.001$ \\
\hline \multicolumn{5}{|l|}{ Autism Diagnostic Interview-Revised* } \\
\hline \multicolumn{5}{|l|}{ Most severe at 4 to $5 \mathrm{yr}$} \\
\hline Social reciprocal interaction & $19.68 \pm 6.98$ & - & - & - \\
\hline Communication: verbal & $14.68 \pm 5.03$ & - & - & - \\
\hline Communication: nonverbal & $7.84 \pm 3.43$ & - & - & - \\
\hline Repetitive or stereotyped behaviour or interests & $7.26 \pm 2.67$ & - & - & - \\
\hline Social reciprocal interaction & $10.29 \pm 4.71$ & - & - & - \\
\hline Repetitive or stereotyped behaviour or interests & $5.27 \pm 2.52$ & - & - & - \\
\hline \multicolumn{5}{|l|}{ Social Responsiveness Scale† } \\
\hline Social awareness deficits & $20.57 \pm 5.29$ & $12.50 \pm 5.95$ & $F=78.05$ & $<0.001$ \\
\hline Social communication deficits & $40.04 \pm 14.95$ & $8.82 \pm 7.09$ & $F=208.91$ & $<0.001$ \\
\hline Social emotion deficits & $12.81 \pm 4.66$ & $3.57 \pm 3.52$ & $F=161.74$ & $<0.001$ \\
\hline Stereotyped behaviours & $20.28 \pm 7.54$ & $3.92 \pm 4.31$ & $F=216.78$ & $<0.001$ \\
\hline Total score & $93.70 \pm 28.74$ & $28.80 \pm 17.11$ & $F=231.99$ & $<0.001$ \\
\hline
\end{tabular}

*Values are mean \pm standard deviation unless otherwise specified.

†The Autism Diagnostic Interview-Revised is an investigator-based interview of the caregiver, and the Social Responsiveness Scale is a caregiver-report measure of autistic traits in natural social settings. The subscores and total scores for both scales are shown here. 
disorder at the most severe age (i.e., 4-5 yr) passed the threshold for a diagnosis of autism. As expected, participants with autism spectrum disorder had more severe current autism symptoms than typically developing controls (as assessed by the SRS).

\section{Brain regions with significant group differences in cortical thickness}

We compared cortical thickness in an age- and sex-comparable subsample aged 8-20 years (autism spectrum disorder: $n=$ 88 , mean age \pm SD $12.7 \pm 3.1 \mathrm{yr}$, male $95.5 \%$; typically developing controls: $n=51$, mean age \pm SD $13.7 \pm 3.6 \mathrm{yr}$, male $88.2 \%$; Table 2). Compared to typically developing controls, participants with autism spectrum disorder showed significantly reduced mean cortical thickness in both hemispheres (Cohen $d=0.403$ for the right hemisphere and 0.360 for the left hemisphere). Nine cortical regions over the right hemisphere showed reduced cortical thickness in participants with autism spectrum disorder after FDR correction: the middle-posterior part of the cingulate gyrus, the posterior transverse collateral sulcus, the orbital gyri, the superior segment of the circular sulcus of the insula, the posterior ramus of the lateral sulcus, the triangular part of the inferior frontal gyrus, the superior temporal sulcus, the orbital part of the inferior frontal gyrus and the lateral occipitotemporal gyrus (Cohen $d=0.358-0.581$; Table 2 and Figure 1). These regions remained significantly different between participants with autism spectrum disorder and typically developing controls when we analyzed the full sample (118 participants with autism spectrum disorder and 122 typically developing controls), controlling for sex, age, IQ, handedness and intracranial volume (Appendix 1, Table S1, available at jpn.ca). Although age was a significant covariate for the thickness of most cortical regions, we found no age $\times$ diagnosis interaction (all $p>0.10)$.

Surprisingly, although some of the left cortical regions were significantly thinner before correction (uncorrected $p<$ 0.05; including the precuneus, the superior occipital sulcus, the transverse occipital sulcus, the superior parietal lobule and the middle-posterior part of the cingulate gyrus and sulcus), none of the findings for these regions survived FDR correction using the classical 1-stage method $\left(q_{\mathrm{FDR}}=0.067\right)$. We repeated the multiple test corrections using another FDR method (graphically sharpened method) and found that the findings for these left cortical regions did survive FDR correction to reveal a thinner cortex $\left(q_{\mathrm{FDR}}=0.035\right.$; Appendix 1, Table S2).

\section{SNP main effects}

As expected, genotype frequency was not associated with diagnosis (Appendix 1, Table S3) for imaging genetic translation in the current sample, which may not have had adequate

Table 2: Group comparison of the cortical thickness of both hemispheres and 9 altered cortical regions ${ }^{\star}$

\begin{tabular}{|c|c|c|c|c|c|c|}
\hline Characteristic & $\begin{array}{l}\text { Autism spectrum disorder† } \\
\qquad n=88\end{array}$ & $\begin{array}{l}\text { Typically developing controls } † \\
\qquad n=51\end{array}$ & $F / \chi^{2} / t$ & $p$ value & $q_{\mathrm{FDR}}$ & Cohen $d$ \\
\hline Age, yr (range) & $12.68 \pm 3.09(8-20)$ & $13.73 \pm 3.61(8-20)$ & $F=3.24$ & 0.07 & - & - \\
\hline Male, $n(\%)$ & $84(95.5)$ & $45(88.2)$ & $\chi^{2}=2.52$ & 0.11 & - & - \\
\hline Left-handedness, $n$ (\%) & $80(90.9)$ & $48(94.1)$ & $\chi^{2}=0.46$ & 0.50 & - & \\
\hline Full-scale IQ & $104 \pm 16$ & $113 \pm 12$ & $F=13.51$ & $<0.001$ & - & - \\
\hline \multicolumn{7}{|l|}{ Cortical thickness, $\mathrm{mm}$} \\
\hline Right hemisphere & $3.101 \pm 0.169$ & $3.157 \pm 0.100$ & $t=-2.65$ & 0.009 & - & 0.403 \\
\hline Left hemisphere & $3.108 \pm 0.165$ & $3.158 \pm 0.107$ & $t=-2.50$ & 0.014 & - & 0.360 \\
\hline $\begin{array}{l}\text { Right middle-posterior part of the } \\
\text { cingulate gyrus and sulcus } \\
\text { (posterior midcingulate cortex) }\end{array}$ & $3.383 \pm 0.223$ & $3.498 \pm 0.169$ & $t=-3.61$ & $<0.001$ & 0.004 & 0.581 \\
\hline $\begin{array}{l}\text { Right posterior transverse } \\
\text { collateral sulcus }\end{array}$ & $2.730 \pm 0.285$ & $2.855 \pm 0.246$ & $t=-3.39$ & 0.001 & 0.004 & 0.470 \\
\hline Right orbital gyri & $3.399 \pm 0.219$ & $3.500 \pm 0.159$ & $t=-2.93$ & 0.004 & 0.008 & 0.528 \\
\hline $\begin{array}{l}\text { Right superior segment of the } \\
\text { circular sulcus of the insula }\end{array}$ & $3.424 \pm 0.227$ & $3.508 \pm 0.169$ & $t=-3.22$ & 0.002 & 0.005 & 0.420 \\
\hline $\begin{array}{l}\text { Right posterior ramus of the lateral } \\
\text { sulcus }\end{array}$ & $3.181 \pm 0.239$ & $3.294 \pm 0.194$ & $t=-2.76$ & 0.007 & 0.008 & 0.519 \\
\hline $\begin{array}{l}\text { Right triangular part of the inferior } \\
\text { frontal gyrus }\end{array}$ & $3.281 \pm 0.265$ & $3.380 \pm 0.197$ & $t=-2.82$ & 0.006 & 0.008 & 0.424 \\
\hline $\begin{array}{l}\text { Right superior temporal sulcus } \\
\text { (parallel sulcus) }\end{array}$ & $3.156 \pm 0.202$ & $3.222 \pm 0.165$ & $t=-2.73$ & 0.007 & 0.008 & 0.358 \\
\hline $\begin{array}{l}\text { Right orbital part of the inferior } \\
\text { frontal gyrus }\end{array}$ & $3.409 \pm 0.301$ & $3.521 \pm 0.226$ & $t=-2.90$ & 0.004 & 0.008 & 0.421 \\
\hline $\begin{array}{l}\text { Right lateral occipitotemporal } \\
\text { gyrus (fusiform gyrus, O4-T4) }\end{array}$ & $3.408 \pm 0.211$ & $3.504 \pm 0.179$ & $t=-2.61$ & 0.010 & 0.010 & 0.491 \\
\hline
\end{tabular}

FDR $=$ false discovery rate.

${ }^{*}$ The comparison of cortical thickness used age, sex, full-scale IQ and intracranial volume as covariates. Multiple comparisons of the 9 regions were adjusted using false discovery rate. †Values are mean \pm standard deviation unless otherwise specified. 
A
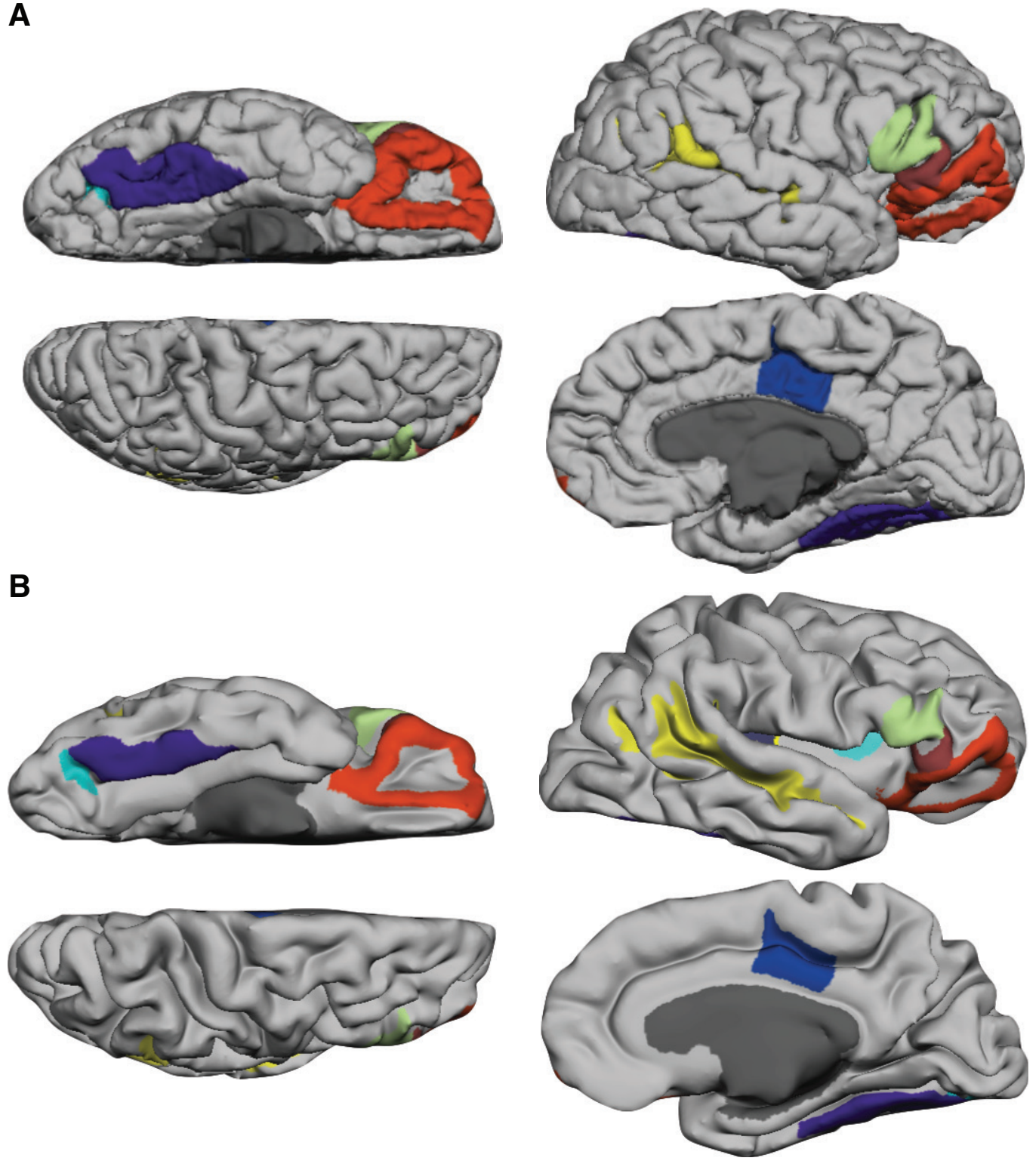

Right orbital gyri
Right lateral occipitotemporal gyrus (fusiform gyrus, O4-T4)
Right triangular part of the inferior frontal gyrus
Right orbital part of the inferior frontal gyrus
Right superior temporal sulcus (parallel sulcus)
Right middle-posterior part of the cingulate gyrus and sulcus (posterior midcingulate cortex)
Right posterior transverse collateral sulcus
Right superior segment of the circular sulcus of the insula
Right posterior ramus of the lateral sulcus

Figure 1: The 9 cortical regions identified with reduced cortical thickness, presented on $(A)$ a standard brain cortex and (B) an inflated brain cortex. 
power to detect a genetic association. Therefore, in the following analysis we evaluated the effects of SNPs and diagnosis in a pooled sample.

For mean thickness of the left or right hemisphere and each of the 9 cortical regions identified in our first step, we examined the main effect of each SNP on cortical thickness in the full sample. We summarized the thickness of the above cortical regions for each genotype of the WNT2 SNPs (Appendix 1, Table S4). None of the WNT2 variants showed a main effect on any of the hemispheres or 9 cortical regions. This result was consistent across dominant, recessive and overdominant models (Appendix 1, Table S5).

\section{Interaction between SNP effect and diagnosis}

For each SNP, we examined the interactions between SNP and diagnosis on the cortical thickness of each hemisphere and cortical region in a single-regression model. After FDR correction, we found that rs6950765 had a significant interaction with autism spectrum disorder diagnosis for the cortical thickness of the posterior ramus of the right lateral sulcus $\left(F=10.37, p=0.001, q_{\mathrm{FDR}}=0.004\right)$. Specifically, typically developing controls who carried the GG $(3.17 \pm 0.19 \mathrm{~mm})$ or GC $(3.25 \pm 0.19 \mathrm{~mm})$ genotype had a thinner cortex than those who carried the CC genotype $(3.34 \pm 0.21 \mathrm{~mm} ; F=5.02, p=$ $0.008)$; we did not find the same association in participants with autism spectrum disorder $(F=1.43, p=0.24)$. We repeated this analysis in the 3 genetic models, and the finding for rs6950765 remained in the dominant $\left(q_{\mathrm{FDR}}=0.008\right)$ and overdominant models $\left(q_{\mathrm{FDR}}=0.009\right)$. As well, rs2896218 showed a significant interaction with autism spectrum diagnosis for the triangular part of the right inferior frontal gyrus in the overdominant model $(F=7.42, p=0.007)$, but this finding did not survive FDR correction $\left(q_{\mathrm{FDR}}=0.06\right)$.

\section{Interaction between SNP effect and age}

We examined the interactions between age and SNP on the cortical thickness of each hemisphere and cortical region for each SNP in a single-regression model. In the full sample (combining participants with autism spectrum disorder and typically developing controls), we found main effects of rs6950765 (left side: $F=6.79, p=0.001$; right side: $F=6.94$, $p=0.001$ ) and rs2896218 (left side: $F=5.32, p=0.006$; right side: $F=5.38, p=0.005)$ on the mean thickness of both hemispheres (Figure 2 and Table 3 ), the right middleposterior part of the cingulate gyrus and sulcus, the orbital gyri, the superior segment of the circular sulcus of the insula, the triangular part of the inferior frontal gyrus, and the superior temporal sulcus (Table 3). We also found significant interactions between age and the 2 SNPs in most of these regions (maximal $\mid$ Cohen $d \mid=0.486$; Table 3). Specifically, both SNPs interacted with age on the right middleposterior part of the cingulate gyrus and sulcus, the orbital gyri, the triangular part of the inferior frontal gyrus and the superior temporal sulcus (Table 3 and Appendix 1, Figure S1 and Figure S2 for rs6950765 and rs2896218, respectively). After FDR correction, the SNP $\times$ age interactions remained significant for both SNPs for the middle-posterior part of the cingulate gyrus and sulcus (rs6950765: $q_{\mathrm{FDR}}=0.0002$; rs2896218: $\left.q_{\mathrm{FDR}}=0.004\right)$, the orbital gyri $\left(\mathrm{rs6950765:} q_{\mathrm{FDR}}=\right.$ 0.021 ; rs2896218: $\left.q_{\mathrm{FDR}}=0.046\right)$ and the superior temporal sulcus (rs6950765: $q_{\mathrm{FDR}}=0.023 ;$ rs2896218: $q_{\mathrm{FDR}}=0.046$; Appendix 1, Figure S1 and Figure S2). Our further examination of the SNP $\times$ age interactions in 3 genetic models revealed that these significant findings largely remained in the overdominant model but not in the dominant or recessive models (Appendix 1, Figure S6).

Considering that the distribution of cortical thickness may not have been the same between participants with autism spectrum disorder and typically developing controls, we further examined SNP $\times$ age interactions, stratifying the 2 groups for the 2 SNPs (Appendix 1, Figure S7).

In typically developing controls, the rs $2896218 \times$ age interaction was significant in the right hemisphere $(F=5.30, p=$ $0.006)$, but the interaction term was only nominally significant in the left hemisphere $(F=4.08, p=0.020)$. The rs6950765 $\times$ age interaction was marginally significant in both the left and right hemispheres (left side: $F=4.05, p=0.020$; right side: $F=0.065, p=0.033$ ). For the 9 cortical regions, the rs $2896218 \times$ age interaction was significant in the middle-posterior part of the cingulate gyrus and sulcus $\left(q_{\mathrm{FDR}}=0.003\right)$ and the orbital gyri $\left(q_{\mathrm{FDR}}=0.008\right)$, but the rs6950765 $\times$ age interaction was significant only for the right middle-posterior part of the cingulate gyrus $\left(q_{\mathrm{FDR}}=0.003\right)$.

In autism spectrum disorder, none of the interactions survived FDR correction, though rs6950765 still showed a main effect in the left ( $p=0.004)$ and right hemispheres $(p=0.005)$, as well as in the right middle-posterior part of the cingulate gyrus and sulcus $\left(q_{\mathrm{FDR}}=0.023\right)$ and the superior temporal sulcus $\left(q_{\mathrm{FDR}}=0.023\right)$ after controlling for their interaction with age.

Examination of the SNP main effect and SNP $\times$ age interaction in the 3 genetic models (Appendix 1, Table S8) showed that the above findings mostly remained in the overdominant and recessive models but not the dominant model in typically developing controls. In autism spectrum disorder, rs6950765 and rs2896218 showed significant main effects in both hemispheres in the overdominant model after controlling for their interactions with age; rs6950765 also showed a main effect on the superior temporal sulcus in the overdominant model $\left(q_{\mathrm{FDR}}=0.023\right)$. In autism spectrum disorder, the rs6950765 $\times$ age interaction was significant in both hemispheres in both the overdominant and recessive models. For the 9 regions, rs6950765 showed a significant interaction with age on the thickness of the right middle-posterior part of the cingulate gyrus and sulcus $\left(q_{\mathrm{FDR}}=0.037\right)$ and right orbital gyrus $\left(q_{\mathrm{FDR}}=0.037\right)$ in the recessive model, and this finding survived FDR correction (Appendix 1, Table S8). None of the other SNP $\times$ age interactions survived FDR correction.

We also analyzed the genetic association between the WNT2 SNPs and cortical thickness across the 148 regions (74 for each hemisphere) of the whole brain, including SNP main effect, $\mathrm{SNP} \times$ diagnosis interaction and $\mathrm{SNP} \times$ age interaction (Appendix 1, Table S9). 


\section{Discussion}

As one of the first studies to explore the effect of WNT2 variants on cortical thickness, the current study had 2 main findings. First, we found a reduction of mean cortical thickness in both hemispheres in people with autism spectrum disorder compared to typically developing controls. As well, 9 regions in the right hemisphere showed significantly lower cortical thickness in participants with autism spectrum disorder after FDR correction. Second, 2 SNPs of WNT2 (rs6950765 and rs2896218) showed significant interactions with age on the mean cortical thickness of both hemispheres and several cortical regions that were altered in autism spectrum disorder (the middle-posterior part of the cingulate gyrus and sulcus, the orbital gyri and the superior temporal sulcus).

Consistent with several studies, $3,5,48,49$ our findings suggest a general reduction in cortical thickness in both hemispheres in people with autism spectrum disorder. In the present study, the 9 regions that showed significantly reduced thickness in participants with autism spectrum disorder were located mainly in the right cingulate gyrus, the orbital gyrus, the insula, the inferior frontal gyrus (orbital part and triangular part) and the lateral occipitotemporal gyrus. Several of these regions overlapped with previous findings that showed thinner cortex in regions of the frontal, ${ }^{5,49}$ temporal $^{2,48}$ and occipital areas $^{5}$ in people with autism spectrum disorder of a similar age range, and in studies revealing an atypical trajectory of the frontal and temporal cortices in longitudinal studies ranging from childhood ${ }^{7}$ to young adulthood. ${ }^{1}$ However, our findings of a thinner cortex were inconsistent with 2 recent large-scale studies that reported increased thickness over the frontal cortex but contradictory findings for the temporal cortex..$^{2,8}$ It should be noted that the quality-control methods, analysis pipelines (CIVET v. FreeSurfer) and segmentation or parcellation methods varied across these studies.
A

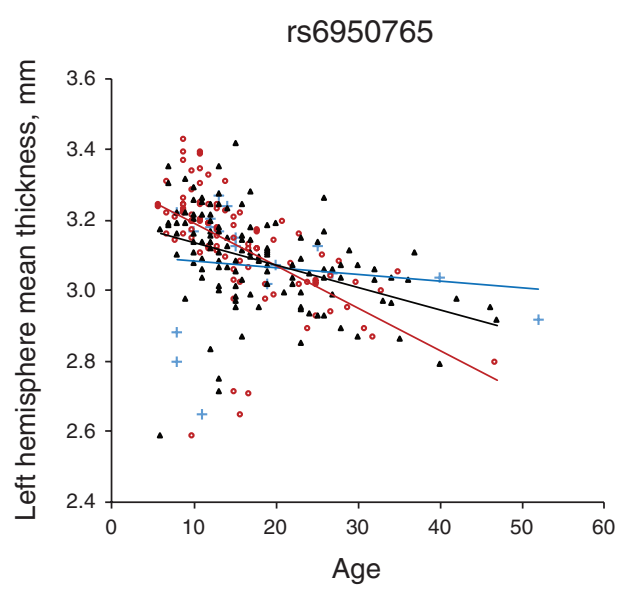

C

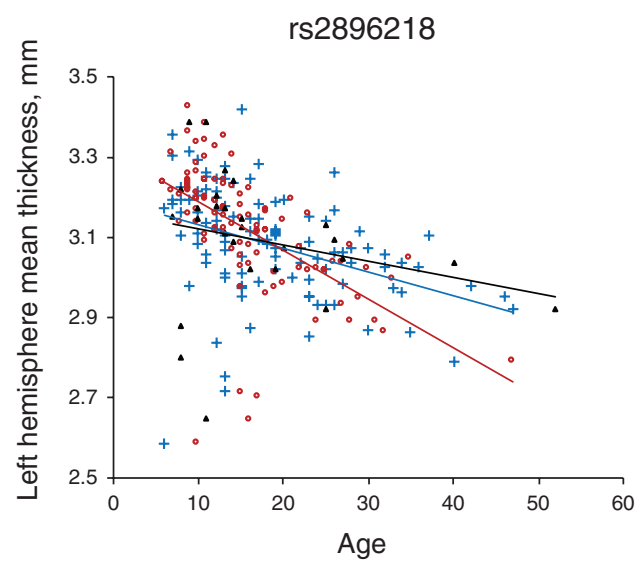

B

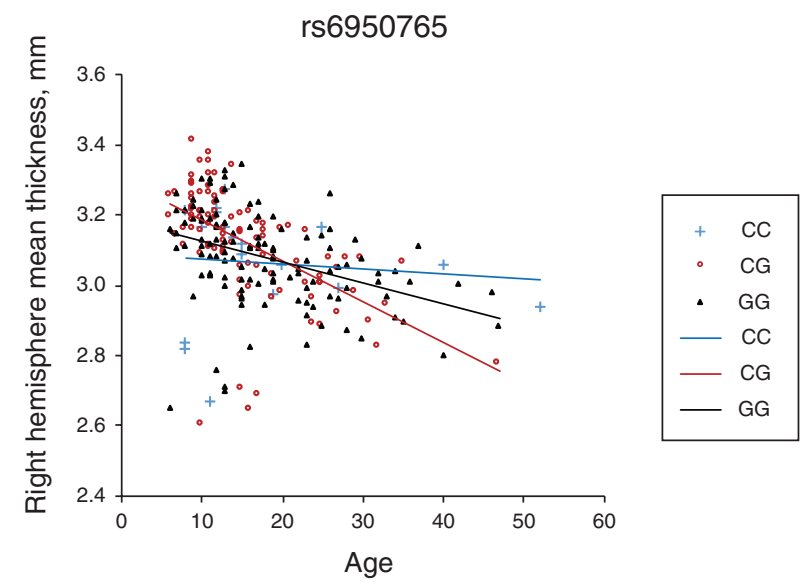

D

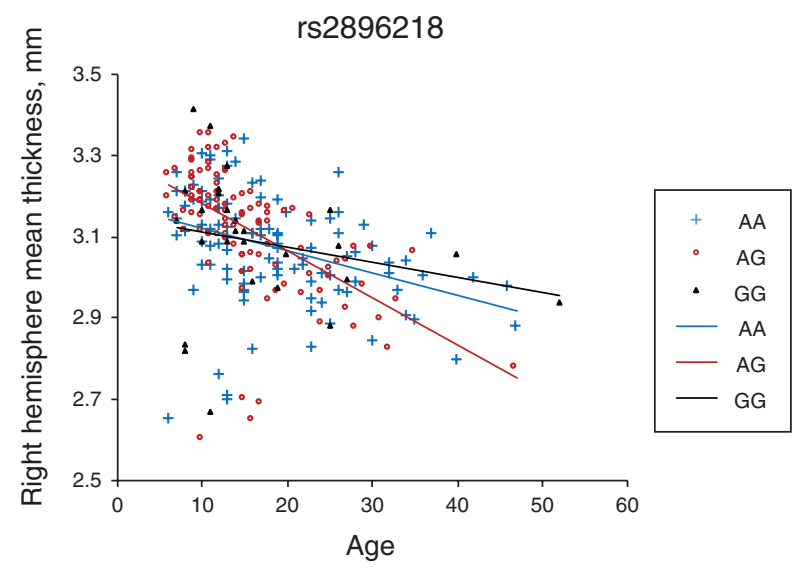

Figure 2: The genotypes rs6950765 and rs2896218 were associated with mean cortical thickness in both hemispheres. (A) Genotype rs6950765 and the mean thickness of the left hemisphere. (B) Genotype rs6950765 and the mean thickness of the right hemisphere. (C) Genotype rs2896218 and the mean thickness of the left hemisphere. (D) Genotype rs2896218 and the mean thickness of the right hemisphere. The CG of rs6950765 and the AG of rs2896218 showed greater cortical thinning of both hemispheres with age. 
To complicate matters, heterogeneity remains an unresolved issue in neuroimaging research in people autism spectrum disorder. For example, samples composed of different developmental stages, sexes, clinical severities and comorbidities could have contributed to inconsistent findings. Our sample (aged 6-56 years) covered younger childhood $^{8}$ and the entire period of adolescence, ${ }^{2}$ which were assumed to show greater alterations in cortical thickness, but unlike the wide age range of large-scale studies, ${ }^{2,8}$ we were unable to capture early developmental changes before age
6 years, the stage when accelerated cortical expansion has been reported.

In addition, some regions have been rarely reported in terms of cortical thickness in autism spectrum disorder (e.g., the insula), although evidence has suggested altered volume or function in these regions in people with autism spectrum disorder. ${ }^{50-53}$ Notably, previous studies have shown reduced cortical thickness in the left hemisphere in children ${ }^{54,55}$ or adults $^{55,56}$ with autism spectrum disorder. Our findings in a larger sample of participants with autism spectrum disorder

Table 3: Significant main effect of SNPs and age $\times$ SNP interactions in the entire sample $(n=240)$

\begin{tabular}{|c|c|c|c|c|c|}
\hline \multirow[b]{2}{*}{ Region } & \multicolumn{2}{|c|}{ Main effect of SNP } & \multicolumn{3}{|c|}{ Age $\times$ SNP interaction } \\
\hline & Cohen $d$ & Statistics & Cohen $d$ & Statistics & $R^{2}$ \\
\hline \multicolumn{6}{|l|}{ rs2896218 } \\
\hline $\begin{array}{l}\text { Left hemisphere } \\
\text { (mean thickness) }\end{array}$ & $\begin{array}{l}\text { AA v. GG: } 0.038 \\
\text { AG v. GG: } 0.175\end{array}$ & $\begin{array}{c}F=5.32 \\
p=0.006\end{array}$ & $\begin{array}{l}\text { Age } \times \text { AA: }-0.316 \\
\text { Age } \times \text { AG: }-0.475 \\
\text { Age } \times \text { GG: }-0.127\end{array}$ & $\begin{array}{c}F=5.06 \\
p=0.007\end{array}$ & 0.27 \\
\hline $\begin{array}{l}\text { Right hemisphere } \\
\text { (mean thickness) }\end{array}$ & $\begin{array}{l}\text { AA v. GG: } 0.034 \\
A G \text { v. GG: } 0.174\end{array}$ & $\begin{array}{c}F=5.38 \\
p=0.005\end{array}$ & $\begin{array}{l}\text { Age } \times \text { AA: }-0.300 \\
\text { Age } \times \text { AG: }-0.459 \\
\text { Age } \times \text { GG: }-0.122\end{array}$ & $\begin{array}{l}F=4.78 \\
p=0.009\end{array}$ & 0.26 \\
\hline $\begin{array}{l}\text { Right middle-posterior part } \\
\text { of the cingulate gyrus and } \\
\text { sulcus (posterior } \\
\text { midcingulate cortex) }\end{array}$ & $\begin{array}{l}\text { AA v. GG: } 0.152 \\
\text { AG v. GG: } 0.283\end{array}$ & $\begin{array}{c}F=10.33 \\
p<0.001^{\star \star}\end{array}$ & $\begin{array}{c}\text { Age } \times \text { AA: }-0.273 \\
\text { Age } \times \text { AG: }-0.437 \\
\text { Age } \times \text { GG: } 0.008\end{array}$ & $\begin{array}{c}F=9.71 \\
p<0.001^{\star *}\end{array}$ & 0.24 \\
\hline Right orbital gyri & $\begin{array}{l}\text { AA v. GG: } 0.098 \\
\text { AG v. GG: } 0.183\end{array}$ & $\begin{array}{c}F=4.31 \\
p=0.015\end{array}$ & $\begin{array}{l}\text { Age } \times \text { AA: }-0.320 \\
\text { Age } \times \text { AG: }-0.386 \\
\text { age } \times \text { GG: }-0.036\end{array}$ & $\begin{array}{c}F=5.27 \\
p=0.006^{\star}\end{array}$ & 0.22 \\
\hline $\begin{array}{l}\text { Right superior segment of } \\
\text { the circular sulcus of the } \\
\text { insula }\end{array}$ & $\begin{array}{l}\text { AA v. GG: } 0.090 \\
A G \text { v. GG: } 0.161\end{array}$ & $\begin{array}{l}F=3.31 \\
p=0.038\end{array}$ & $\begin{array}{l}\text { Age } \times \text { AA: }-0.222 \\
\text { Age } \times \text { AG: }-0.297 \\
\text { Age } \times \text { GG: }-0.042\end{array}$ & $\begin{array}{l}F=2.30 \\
p=0.10\end{array}$ & 0.14 \\
\hline $\begin{array}{l}\text { Right triangular part of the } \\
\text { inferior frontal gyrus }\end{array}$ & $\begin{array}{l}\text { AA v. GG: } 0.025 \\
\text { AG v. GG: } 0.143\end{array}$ & $\begin{array}{c}F=4.10 \\
p=0.018\end{array}$ & $\begin{array}{l}\text { Age } \times \text { AA: }-0.223 \\
\text { Age } \times \text { AG: }-0.380 \\
\text { Age } \times \text { GG: }-0.096\end{array}$ & $\begin{array}{c}F=4.40 \\
p=0.013\end{array}$ & 0.17 \\
\hline $\begin{array}{l}\text { Right superior temporal } \\
\text { sulcus (parallel sulcus) }\end{array}$ & $\begin{array}{l}\text { AA v. GG: }-0.017 \\
\text { AG v. GG: } 0.149\end{array}$ & $\begin{array}{c}F=6.65 \\
p=0.002^{\star}\end{array}$ & $\begin{array}{l}\text { Age } \times \text { AA: }-0.286 \\
\text { Age } \times \text { AG: }-0.465 \\
\text { Age } \times \text { GG: }-0.199\end{array}$ & $\begin{array}{c}F=4.91 \\
p=0.008^{*}\end{array}$ & 0.27 \\
\hline \multicolumn{6}{|l|}{ rs6950765 } \\
\hline $\begin{array}{l}\text { Left hemisphere } \\
\text { (mean thickness) }\end{array}$ & $\begin{array}{l}\text { CC v. GG: }-0.110 \\
\text { CG v. GG: } 0.190\end{array}$ & $\begin{array}{c}F=6.79 \\
p=0.001\end{array}$ & $\begin{array}{l}\text { Age } \times \text { CC: }-0.057 \\
\text { Age } \times \text { CG: }-0.484 \\
\text { Age } \times \text { GG: }-0.344\end{array}$ & $\begin{array}{c}F=6.01 \\
p=0.003\end{array}$ & 0.29 \\
\hline $\begin{array}{l}\text { Right hemisphere } \\
\text { (mean thickness) }\end{array}$ & $\begin{array}{l}\text { CC v. GG: }-0.107 \\
\text { CG v. GG: } 0.196\end{array}$ & $\begin{array}{l}F=6.94 \\
p=0.001\end{array}$ & $\begin{array}{l}\text { Age } \times \text { CC: }-0.050 \\
\text { Age } \times \text { CG: }-0.471 \\
\text { Age } \times \text { GG: }-0.329\end{array}$ & $\begin{array}{c}F=5.91 \\
p=0.003\end{array}$ & 0.27 \\
\hline $\begin{array}{l}\text { Right middle-posterior part } \\
\text { of the cingulate gyrus and } \\
\text { sulcus (posterior } \\
\text { midcingulate cortex) }\end{array}$ & $\begin{array}{l}\text { CC v. GG: }-0.229 \\
\text { CG v. GG: } 0.184\end{array}$ & $\begin{array}{c}F=14.48 \\
p<0.001^{\star \star}\end{array}$ & $\begin{array}{l}\text { Age } \times \text { CC: } 0.089 \\
\text { Age } \times \text { CG: }-0.454 \\
\text { Age } \times \text { GG: }-0.301\end{array}$ & $\begin{array}{c}F=13.97 \\
p<0.001^{\star \star}\end{array}$ & 0.27 \\
\hline Right orbital gyri & $\begin{array}{l}\text { CC v. GG: }-0.157 \\
\text { CG v. GG: } 0.132\end{array}$ & $\begin{array}{c}F=7.04 \\
p=0.001^{*}\end{array}$ & $\begin{array}{l}\text { Age } \times \text { CC: } 0.016 \\
\text { Age } \times \text { CG: }-0.396 \\
\text { Age } \times \text { GG: }-0.334\end{array}$ & $\begin{array}{c}F=7.09 \\
p=0.001^{\star}\end{array}$ & 0.23 \\
\hline $\begin{array}{l}\text { Right superior segment of } \\
\text { the circular sulcus of the } \\
\text { insula }\end{array}$ & $\begin{array}{l}\text { CC v. GG: }-0.151 \\
\text { CG v. GG: } 0.121\end{array}$ & $\begin{array}{c}F=6.26 \\
p=0.002^{*}\end{array}$ & $\begin{array}{l}\text { Age } \times \text { CC: } 0.032 \\
\text { Age } \times \text { CG: }-0.333 \\
\text { Age } \times \text { GG: }-0.234\end{array}$ & $\begin{array}{c}F=5.97 \\
p=0.003^{\star}\end{array}$ & 0.16 \\
\hline $\begin{array}{l}\text { Right triangular part of the } \\
\text { inferior frontal gyrus }\end{array}$ & $\begin{array}{l}\text { CC v. GG: }-0.061 \\
\text { CG v. GG: } 0.193\end{array}$ & $\begin{array}{c}F=6.27 \\
p=0.002^{*}\end{array}$ & $\begin{array}{l}\text { Age } \times \text { CC: }-0.048 \\
\text { Age } \times \text { CG: }-0.379 \\
\text { Age } \times \text { GG: }-0.235\end{array}$ & $\begin{array}{c}F=5.00 \\
p=0.008^{\star}\end{array}$ & 0.18 \\
\hline $\begin{array}{l}\text { Right superior temporal } \\
\text { sulcus (parallel sulcus) }\end{array}$ & $\begin{array}{l}\text { CC v. GG: }-0.075 \\
\text { CG v. GG: } 0.215\end{array}$ & $\begin{array}{c}F=8.01 \\
p<0.001^{\star *}\end{array}$ & $\begin{array}{l}\text { Age } \times \text { CC: }-0.125 \\
\text { Age } \times \text { CG: }-0.486 \\
\text { Age } \times \text { GG: }-0.315\end{array}$ & $\begin{array}{c}F=6.07 \\
p=0.003^{*}\end{array}$ & 0.27 \\
\hline
\end{tabular}

FDR $=$ false discovery rate SNP = single nucleotide polymorphism .

Model: Cortical thickness of specific brain region $=\beta_{0}+\beta_{1}$ "SNP" $+\beta_{2}$ "age $\times$ SNP" $+\beta_{3}$ "age" $+\beta_{4}$ "sex" $+\beta_{5}$ "full-scale IQ" $+\beta_{6}$ "handedness" + $\beta_{7}$ "intracranial volume" $+\varepsilon$. Findings for the main effects of SNP and age $\times$ SNP interaction for rs2896218 and rs6950765 are summarized above. For the 9 cortical regions, ${ }^{*} q_{\mathrm{FDR}}<0.05,{ }^{\star *} q_{\mathrm{FDR}}<0.01$. 
(aged 6-28 yr) did show reduced mean cortical thickness in both hemispheres, but only the 9 cortical regions in the right hemisphere survived FDR correction. Several left cortical regions (i.e., precuneus, superior occipital sulcus and transverse occipital sulcus, superior parietal lobule and middleposterior part of the cingulate gyrus and sulcus) did not survive FDR correction using the classical 1-stage method $\left(q_{\mathrm{FDR}}=0.067\right)$ but did survive when we used a graphically sharpened approach $\left(q_{\mathrm{FDR}}=0.035\right.$; Appendix 1, Table S4), showing a thinner cortex. In this sense, adopting a more stringent method (i.e., the classical 1-stage method) for multiple test corrections suggests that our findings for regions of altered cortical thickness may have been conservative but more robust. Nonetheless, evidence has shown that autism spectrum disorder (aged 2-65 years) is significantly associated with reduced asymmetry of cortical thickness, mainly in the medial frontal, orbitofrontal, cingulate and inferior temporal areas. ${ }^{57}$ Our findings of significantly reduced thickness in the inferior frontal, temporal and cingulate cortices of the right hemisphere may lend further support for asymmetry in cortical thickness in specific cortical regions in people with autism spectrum disorder.

Our novel finding that WNT2 SNPs (rs2896218 and rs6950765) interacted with age over the cortical regions that showed reduced thickness in autism spectrum disorder is intriguing. Previous research has suggested that WNT2 is one of the candidate genes for autism in Asian samples. ${ }^{27}$ Our previous study using a trios design also showed a significant association between autism and a WNT2 haplotype composed of rs2896218 and rs6950765 (G-G), ${ }^{26}$ as well as the associations of these SNPs with language development ( $p=$ $0.0033)^{58}$ and stereotyped behaviours (Cohen $\left.d=-0.44\right) \cdot{ }^{30}$ In the current study, we found that the same 2 SNPs were associated with cortical thinning in the middle-posterior cingulate gyrus and orbital gyri: people carrying heterozygous genotypes of rs6950765 (CG) and rs2896218 (AG) showed a greater magnitude of cortical thinning with age compared to those carrying homozygous genotypes of risk alleles (rs6950765: GG; rs2896218: GG), in support of an overdominant model. It remains to be elucidated whether the exaggerated thinning of the cortex observed in people with specific genotypes of WNT2 is attributable to alterations in cortical dendritic growth and spine formation regulated by WNT2.20-22 On the other hand, the statistical significance of the age $x$ SNP interaction disappeared in the autism spectrum disorder group after FDR correction. Future studies should examine the age $\times$ SNP interaction in large-scale samples of participants with autism spectrum disorder and a wider age range (covering middle and late adulthood) to reveal long-term trends for cortical reduction with age.

The 2 WNT2 SNPs (rs2896218 and rs6950765) located in intron 4 were physically close to the SNPs associated with autism spectrum disorder (i.e., rs377954, rs4727847 and rs3729629) reported by Marui and colleagues, ${ }^{27}$ with a distance of only about 5.4 to $6.7 \mathrm{~kb}$. New evidence emphasizes the intriguing role of noncoding regions in the genome, particularly for regulatory elements. These 2 SNPs were not located at the splicing site of WNT2, and not at a transcription factor binding site. However, in our subsidiary analysis, we explored whether these 2 SNPs might be located at the boundary of topological association domains and close to the predicted CCCTC-binding factor (CTCF) binding sites (http://insulatordb.uthsc.edu/; Appendix 1, Table S10).

CTCF is a versatile transcription regulator that is evolutionarily conserved across species. In the eukaryotic genome, CTCF plays a crucial role in the global organization of chromatin architecture, ${ }^{59}$ including chromatin insulation. ${ }^{59,60}$ Chromatin insulators are enhancer-blocking and domain-bordering, ${ }^{61,62}$ and therefore are critical regulatory elements for the control of gene expression. They represent a class of diverged DNA sequences capable of shielding genes against inappropriate cis-regulatory signals from their genome neighbourhood. Evidence from the Hi-C experiment, a method to study the 3-dimensional architecture of genomes, has demonstrated that interacting genomic regions commonly contain CTCF binding sites and that the boundaries of genomic topological domains are enriched for CTCF binding sites. ${ }^{63-65}$ Whether the CTCF binding sites near rs6950765 and rs2896218 are involved in the regulation of WNT2 gene expression is intriguing. Moreover, emerging studies have highlighted that the Wnt signalling pathway also plays a major role in regulating tolerance versus immunity ${ }^{66}$ and immune response to infection. ${ }^{67}$ Whether such mechanisms are also related to the thinning of cortical structures via CTCF binding sites near rs6950765 and rs2896218 warrants further research.

\section{Limitations}

Despite its strengths (including a 2-stage approach to examine cortical regions relevant to autism spectrum disorder and selecting WNT2 gene variants based on previous findings in the Taiwanese autism spectrum disorder cohort), the present study had several limitations.

We performed statistical analyses on each hemisphere and targeted the 9 regions that were significantly altered in autism spectrum disorder. Although we also analyzed the genetic association of WNT2 variants analyzed for the whole brain (Appendix 1, Table S9), future studies may consider a more advanced data-driven method such as machine learning to investigate the effect of the WNT2 variants on the entire cortex.

The present study was a cross-sectional study, and the associations found between age and cortical thickness need to be examined in longitudinal studies that completely match participants with autism spectrum disorder and typically developing controls based on age and sex to clarify exact age effects in individuals. As well, age trends may differ during the crucial earlier developmental stage before age 6 years, which we did not sample in the present study but should be examined further.

Our sample size may have been insufficient to identify small effect differences, particularly for the interaction terms. Using sensitivity power calculations, our total sample size of 240 had $80 \%$ power to detect the main effect of effect size 0.23 (at $\alpha=0.05$ ), but identifying interactions for the same effect size would need a larger sample to determine. 
Some of the cortical regions we evaluated in the right hemisphere are functionally associated with cognitive and behavioural differences in autism spectrum disorder. Although evidence suggests that cortical thickness later in life is attributable to genetic control, intervention effect and gene-environment interaction on the cortex remain essential factors to be controlled for in future imaging genetic studies. Nevertheless, our translational study combined neuroimaging and genetics at a single research site to examine the genetic association between WNT2 and cortical thickness, and was the first of its kind to explore the genetic effect on changes in cortical thickness over time. Future studies may adopt a trajectory analysis to validate our exploratory findings in larger independent samples.

\section{Conclusion}

This study explored the associations between WNT2 variants and the cortical thickness of brain regions that are altered in autism spectrum disorder, providing new evidence to show that WNT2 variants might affect the thinning of the cortex. If validated, our findings warrant further functional research into how this gene may change the dynamics of the cortex over time using animal studies. The potential role of the topological association domains of WNT2 in the regulation of cortical development is the next focus of interest. Cumulative evidence has suggested the involvement of the immune response in the mechanisms of mental disorders, including autism spectrum disorder, highlighting the interrelationships between the immune and nervous systems. As an exploratory approach to the translational role of WNT2, the results of the current study pave a putative way toward future research into the role of immunological mechanisms in the cortical alterations of autism spectrum disorder.

Acknowledgements: The authors thank the National Center for Genome Medicine of the National Core Facility Program for Biotechnology, Ministry of Science and Technology, for the technical and bioinformatics support. They also thank the Department of Medical Imaging and the Eighth Core Lab, Department of Medical Research, National Taiwan University Hospital, for MRI scanning and technical support, respectively, during the study. This study was supported by grants from the Ministry of Science and Technology [grant numbers NSC99-2321-B-002-037, NSC100-2321-B-002-015, NSC101-2314-B-002136-MY3, MOST102-2314-B-002-019, MOST106-2410-H-002-075-MY2, MOST108-2628-H-002-009-MY3], and National Taiwan University Hospital [grant numbers NTUH101-S1910, NTUH106-N04, NTUH108S4103, NTUH108-S4103, UN109-007], Taiwan. The preparation of the manuscript was supported by the National Health Research Institute, Taiwan [grant number NHRI-EX107-10404PI, NHRI-EX108-10404PI].

Affiliations: From the Department of Psychiatry, National Taiwan University Hospital and College of Medicine, Taipei, Taiwan (Chien, Chen, Chiu, Tsai, Gau); and the Graduate Institute of Clinical Medicine, and Graduate Institute of Brain and Mind Sciences, College of Medicine, National Taiwan University, Taipei, Taiwan (Chen, Gau).

Competing interests: None declared.

Contributors: Y. Chien and S. S. Gau designed the study. Y. Chen, Y. Chien, Y. Chiu and W. Tsai acquired the data, which Y. Chien and Y. Chen analyzed. Y. Chien wrote the article, which Y. Chen, Y. Chiu, W. Tsai and S. S. Gau reviewed. All authors approved the final version to be published and can certify that no other individuals not listed as authors have made substantial contributions to the paper.
Content licence: This is an Open Access article distributed in accordance with the terms of the Creative Commons Attribution (CC BY-NC-ND 4.0) licence, which permits use, distribution and reproduction in any medium, provided that the original publication is properly cited, the use is noncommercial (i.e., research or educational use), and no modifications or adaptations are made. See: https: / / creativecommons.org/licenses/by-nc-nd/4.0/

\section{References}

1. Nunes AS, Vakorin VA, Kozhemiako N, et al. Atypical age-related changes in cortical thickness in autism spectrum disorder. Sci Rep 2020;10:11067.

2. van Rooij D, Anagnostou E, Arango C, et al. Cortical and subcortical brain morphometry differences between patients with autism spectrum disorder and healthy individuals across the lifespan: results from the ENIGMA ASD Working Group. Am J Psychiatry 2018;175:359-69.

3. Baribeau DA, Anagnostou E. A comparison of neuroimaging findings in childhood onset schizophrenia and autism spectrum disorder: a review of the literature. Front Psychiatry 2013;4:175.

4. Courchesne E, Karns CM, Davis HR, et al. Unusual brain growth patterns in early life in patients with autistic disorder: an MRI study. Neurology 2001;57:245-54.

5. Zielinski BA, Prigge MB, Nielsen JA, et al. Longitudinal changes in cortical thickness in autism and typical development. Brain 2014;137:1799-812.

6. Wallace GL, Dankner N, Kenworthy L, et al. Age-related temporal and parietal cortical thinning in autism spectrum disorders. Brain 2010;133:3745-54.

7. Yang DY, Beam D, Pelphrey KA, et al. Cortical morphological markers in children with autism: a structural magnetic resonance imaging study of thickness, area, volume, and gyrification. Mol Autism 2016;7:11.

8. Bedford SA, Park MTM, Devenyi GA, et al. Large-scale analyses of the relationship between sex, age and intelligence quotient heterogeneity and cortical morphometry in autism spectrum disorder. Mol Psychiatry 2020;25:614-28.

9. Chen $\mathrm{CH}$, Fiecas M, Gutierrez ED, et al. Genetic topography of brain morphology. Proc Natl Acad Sci U S A 2013;110:17089-94.

10. Valk SL, Xu T, Margulies DS, et al. Shaping brain structure: genetic and phylogenetic axes of macroscale organization of cortical thickness. Sci Adv 2020;6:eabb3417.

11. Chen $\mathrm{CH}$, Gutierrez ED, Thompson $\mathrm{W}$, et al. Hierarchical genetic organization of human cortical surface area. Science 2012;335:1634-6.

12. Chen CH, Panizzon MS, Eyler LT, et al. Genetic influences on cortical regionalization in the human brain. Neuron 2011;72:537-44.

13. Fjell AM, Grydeland H, Krogsrud SK, et al. Development and aging of cortical thickness correspond to genetic organization patterns. Proc Natl Acad Sci U S A 2015;112:15462-7.

14. Udden J, Snijders TM, Fisher SE, et al. A common variant of the CNTNAP2 gene is associated with structural variation in the left superior occipital gyrus. Brain Lang 2017;172:16-21.

15. Zhu B, Chen C, Xue G, et al. Associations between the CNTNAP2 gene, dorsolateral prefrontal cortex, and cognitive performance on the Stroop task. Neuroscience 2017;343:21-9.

16. Clemm von Hohenberg C, Wigand MC, Kubicki M, et al. CNTNAP2 polymorphisms and structural brain connectivity: a diffusiontensor imaging study. J Psychiatr Res 2013;47:1349-56.

17. Dennis EL, Jahanshad N, Rudie JD, et al. Altered structural brain connectivity in healthy carriers of the autism risk gene, CNTNAP2. Brain Connect 2011;1:447-59.

18. Rakic P. Defects of neuronal migration and the pathogenesis of cortical malformations. Prog Brain Res 1988;73:15-37.

19. Huttenlocher PR. Morphometric study of human cerebral cortex development. Neuropsychologia 1990;28:517-27.

20. Okerlund ND, Cheyette BN. Synaptic Wnt signaling-a contributor to major psychiatric disorders? J Neurodev Disord 2011;3:162-74.

21. Steinecke A, Gampe C, Nitzsche F, et al. Drosoph Inf ServC1 knockdown impairs the tangential migration of cortical interneurons by affecting the actin cytoskeleton. Front Cell Neurosci 2014;8:190. 
22. Hiester BG, Galati DF, Salinas PC, et al. Neurotrophin and Wnt signaling cooperatively regulate dendritic spine formation. Mol Cell Neurosci 2013;56:115-27.

23. Ishizuka K, Kamiya A, Oh EC, et al. Drosoph Inf ServC1-dependent switch from progenitor proliferation to migration in the developing cortex. Nature 2011;473:92-6.

24. Schneider T, Przewlocki R. Behavioral alterations in rats prenatally exposed to valproic acid: animal model of autism. Neuropsychopharmacology 2005;30:80-9.

25. Wang Z, Xu L, Zhu X, et al. Demethylation of specific Wnt/betacatenin pathway genes and its upregulation in rat brain induced by prenatal valproate exposure. Anat Rec (Hoboken) 2010;293:1947-53.

26. Chien YL, Wu YY, Chiu YN, et al. Association study of the CNS patterning genes and autism in Han Chinese in Taiwan. Prog Neuropsychopharmacol Biol Psychiatry 2011;35:1512-7.

27. Marui T, Funatogawa I, Koishi S, et al. Association between autism and variants in the wingless-type MMTV integration site family member 2 (WNT2) gene. Int J Neuropsychopharmacol 2010;13:443-9.

28. Wassink TH, Piven J, Vieland VJ, et al. Evidence supporting WNT2 as an autism susceptibility gene. Am J Med Genet 2001;105:406-13.

29. McCoy PA, Shao Y, Wolpert CM, et al. No association between the WNT2 gene and autistic disorder. Am J Med Genet 2002;114:106-9.

30. Chien $\mathrm{YL}, \mathrm{Wu} \mathrm{SW}$, $\mathrm{Chu} \mathrm{CP}$, et al. Attenuated contact heat-evoked potentials associated with sensory and social-emotional symptoms in individuals with autism spectrum disorder. Sci Rep 2017;7:36887.

31. American Psychiatric Association. Diagnostic and statistical manual of mental disorders. Fifth edition. Arlington (VA): American Psychiatric Association Publishing; 2013.

32. Lord C, Rutter M, Le Couteur A. Autism Diagnostic Interview Revised: a revised version of a diagnostic interview for caregivers of individuals with possible pervasive developmental disorders. J Autism Dev Disord 1994;24:659-85.

33. Chen YL, Shen LJ, Gau SS. The Mandarin version of the KiddieSchedule for Affective Disorders and Schizophrenia-Epidemiological version for DSM-5-a psychometric study. J Formos Med Assoc 2017;116:671-8

34. Chien WH, Wu YY, Gau SSF, et al. Association study of the SLC25A12 gene and autism in Han Chinese in Taiwan. Prog Neuropsychopharmacol Biol Psychiatry 2010;34:189-92.

35. Gau SS, Liu LT, Wu YY, et al. Psychometric properties of the Chinese version of the social responsiveness scale. Res Autism Spectr Disord 2013;7:349-60.

36. Xu H, Gregory SG, Hauser ER, et al. SNPselector: a web tool for selecting SNPs for genetic association studies. Bioinformatics 2005; 21:4181-6

37. Dale AM, Fischl B, Sereno MI. Cortical surface-based analysis I. Segmentation and surface reconstruction. Neuroimage 1999;9:179-94.

38. Fischl B. FreeSurfer. Neuroimage 2012;62:774-81.

39. Fischl B, Salat DH, Busa E, et al. Whole brain segmentation: automated labeling of neuroanatomical structures in the human brain Neuron 2002;33:341-55.

40. Fischl B, van der Kouwe A, Destrieux C, et al. Automatically parcellating the human cerebral cortex. Cereb Cortex 2004;14:11-22.

41. Rosen AFG, Roalf DR, Ruparel K, et al. Quantitative assessment of structural image quality. Neuroimage 2018;169:407-18.

42. Desikan RS, Segonne F, Fischl B, et al. An automated labeling system for subdividing the human cerebral cortex on MRI scans into gyral based regions of interest. Neuroimage 2006;31:968-80.

43. Fischl B, Dale AM. Measuring the thickness of the human cerebral cortex from magnetic resonance images. Proc Natl Acad Sci U S A 2000;97:11050-5.

44. Han X, Jovicich J, Salat D, et al. Reliability of MRI-derived measurements of human cerebral cortical thickness: the effects of field strength, scanner upgrade and manufacturer. Neuroimage 2006;32: $180-94$.
45. Zhao K, Liu H, Yan R, et al. Cortical thickness and subcortical structure volume abnormalities in patients with major depression with and without anxious symptoms. Brain Behav 2017;7:e00754.

46. Stephen R, Liu Y, Ngandu T, et al. Brain volumes and cortical thickness on MRI in the Finnish Geriatric Intervention Study to Prevent Cognitive Impairment and Disability (FINGER). Alzheimers Res Ther 2019;11:53.

47. Sanchis-Segura C, Ibanez-Gual MV, Aguirre N, et al. Author correction. Effects of different intracranial volume correction methods on univariate sex differences in grey matter volume and multivariate sex prediction. Sci Rep 2020;10:18937.

48. Hardan AY, Muddasani S, Vemulapalli M, et al. An MRI study of increased cortical thickness in autism. Am J Psychiatry 2006;163:1290-2.

49. Hadjikhani N, Joseph RM, Snyder J, et al. Anatomical differences in the mirror neuron system and social cognition network in autism. Cereb Cortex 2006;16:1276-82.

50. Caria A, de Falco S. Anterior insular cortex regulation in autism spectrum disorders. Front Behav Neurosci 2015;9:38.

51. Odriozola P, Uddin LQ, Lynch CJ, et al. Insula response and connectivity during social and non-social attention in children with autism. Soc Cogn Affect Neurosci 2016;11:433-44.

52. Wang Q, Zhang Z, Dong F, et al. Anterior insula GABA levels correlate with emotional aspects of empathy: a proton magnetic resonance spectroscopy study. PLoS One 2014;9:e113845.

53. Muller-Pinzler L, Krach S, Kramer UM, et al. The social neuroscience of interpersonal emotions. Curr Top Behav Neurosci 2017;30:241-56.

54. Mak-Fan KM, Taylor MJ, Roberts W, et al. Measures of cortical grey matter structure and development in children with autism spectrum disorder. J Autism Dev Disord 2012;42:419-27.

55. Raznahan A, Toro R, Daly E, et al. Cortical anatomy in autism spectrum disorder: an in vivo MRI study on the effect of age. Cereb Cortex 2010;20:1332-40.

56. Scheel C, Rotarska-Jagiela A, Schilbach L, et al. Imaging derived cortical thickness reduction in high-functioning autism: key regions and temporal slope. Neuroimage 2011;58:391-400.

57. Postema MC, van Rooij D, Anagnostou E, et al. Altered structural brain asymmetry in autism spectrum disorder in a study of 54 datasets. Nat Commun 2019;10:4958.

58. Lin PI, Chien YL, Wu YY, et al. The WNT2 gene polymorphism associated with speech delay inherent to autism. Res Dev Disabil 2012;33:1533-40.

59. Phillips JE, Corces VG. CTCF: master weaver of the genome. Cell 2009;137:1194-211.

60. Ohlsson R, Renkawitz R, Lobanenkov V. CTCF is a uniquely versatile transcription regulator linked to epigenetics and disease. Trends Genet 2001;17:520-7.

61. Bell AC, West AG, Felsenfeld G. Insulators and boundaries: versatile regulatory elements in the eukaryotic genome. Science 2001; 291:447-50.

62. West AG, Gaszner M, Felsenfeld G. Insulators: many functions, many mechanisms. Genes Dev 2002;16:271-88.

63. Botta M, Haider S, Leung IX, et al. Intra- and inter-chromosomal interactions correlate with CTCF binding genome wide. Mol Syst Biol 2010;6:426.

64. Dixon JR, Selvaraj S, Yue F, et al. Topological domains in Mamm genomes identified by analysis of chromatin interactions. Nature 2012;485:376-80.

65. Lieberman-Aiden E, van Berkum NL, Williams L, et al. Comprehensive mapping of long-range interactions reveals folding principles of the human genome. Science 2009;326:289-93.

66. Swafford D, Manicassamy S. Wnt signaling in dendritic cells: its role in regulation of immunity and tolerance. Discov Med 2015;19:303-10.

67. Gatica-Andrades M, Vagenas D, Kling J, et al. WNT ligands contribute to the immune response during septic shock and amplify endotoxemia-driven inflammation in mice. Blood Adv 2017;1:1274-86. 